\title{
Mitochondrial-DNA-associated TLR9 signalling is a potential serological biomarker for non-small cell lung cancer
}

\author{
YU-HENG LAI ${ }^{1}$, HAO-YUN LIU ${ }^{2}$, CHUN-YAO HUANG ${ }^{3}$, YAT-PANG CHAU ${ }^{4}$ and SEMON WU ${ }^{5}$ \\ ${ }^{1}$ Department of Chemistry, Chinese Culture University, Taipei 11114; ${ }^{2}$ Institute of Anatomy and Cell Biology, School of Medicine, \\ National Yang Ming University, Taipei 112; ${ }^{3}$ Department of Pulmonary and Critical Care, Taipei Tzu Chi Hospital, \\ The Buddhist Tzu Chi Medical Foundation, New Taipei 231; ${ }^{4}$ Department of Medicine, Mackay Medical College, \\ New Taipei 252; ${ }^{5}$ Department of Life Science, Chinese Culture University, \\ Taipei 11114, Taiwan, R.O.C.
}

Received March 13, 2018; Accepted October 30, 2018

DOI: $10.3892 /$ or.2018.6855

\begin{abstract}
Mitochondrial (mt) DNA has been long suggested to contribute to carcinogenesis, and a variety of mutations in mtDNA have been confirmed to be related to various early stages of cancers; these data revealed that the detection of mtDNA in clinical samples may be a promising approach for cancer diagnosis. In the present study, the serum mtDNA in healthy donors and groups of patients with cancer was detected. It was revealed that patients with lung cancer without metastasis had more mtDNA in serum compared to patients with metastasis. Moreover, TLR9-associated signalling was activated in vitro after treatment with a synthetic $\mathrm{CpG}$ oligodeoxyribonucleotide (ODN) called ODN-M362. In addition, our data revealed that TLR9 and its adaptor protein, MyD88, were induced by ODN-M362 in a dose-dependent manner. A human cytokine array to evaluate stimulation of cytokine secretion by ODN-M362 was also used. Our findings may identify the role that TLR9 and mtDNA play in lung cancer progression and metastasis.
\end{abstract}

\section{Introduction}

Lung cancer has been identified as the most lethal malignancy in the past decades. Among cancers, non-small cell lung cancer (NSCLC) is considered a complex malignancy owing to its diverse alterations in molecular and genomic pathways. Early diagnosis is crucial for increasing the chance for survival;

Correspondence to: Professor Yat-Pang Chau, Department of Medicine, Mackay Medical College, No. 46, Sec. 3, Zhongzheng Road, Sanzhi, New Taipei 252, Taiwan, R.O.C.

E-mail: leonchau@mmc.edu.tw

Professor Semon Wu, Department of Life Science, Chinese Culture University, 55 Hwa-Kang Road, Yang-Ming-Shan, Taipei 11114, Taiwan, R.O.C.

E-mail: semonwu@yahoo.com.tw

Key words: mtDNA, CpG ODN-M362, TLR9, lung cancer however, no highly sensitive and effective biomarker for NSCLC has been validated in clinical applications. Therefore, a potential biomarker that is specific and early for cancer screening is always a perpetual goal for scientists.

Human mitochondrial DNA (mtDNA), a 16,569 kb circular DNA that contains 37 genes, encodes $12 \mathrm{~S}$ and 16S rRNAs, 22 tRNAs, and 13 polypeptides (1). It contains a non-coding circular loop (D-loop) with a large number of unmethylated dinucleotide fragments called CpG islands (2). Since 1973, mtDNA has been suggested to contribute to carcinogenesis (3). Structural differences in mitochondria between patients with cancer and normal controls, observed under electron microscopy, indicated that mitochondrial instability is cancer-associated (4). For example, deletion and insertions within the mtDNA D-loop were observed in primary breast cancer (5). An aberrant mtDNA copy number was also highly associated with lung cancer in a dose-dependent manner (6). Moreover, through comprehensive resequencing microarrays, a wide variety of mutations in mtDNA have been confirmed to be related to early stages of cancers which suggests that analysis of mtDNA in clinical samples may be a potential approach to cancer diagnosis (7).

Recent evidence has revealed that mtDNA participates in a myriad of immune responses. It serves as an effector of pattern recognition receptor (PRR) signalling that triggers the innate immune system to respond to cellular damage, stress, and infection by pathogens (8). One of PRRs, Toll-like receptor (TLR), is activated by mtDNA and induces a signalling cascade that ultimately results in an inflammatory response involving cytokines and other downstream effectors (9). Among the TLRs, TLR1, TLR2, TLR4, TLR5 and TLR6 are activated by bacterial and fungal cell surface molecules, whereas TLR3, TLR7, TLR8 and TLR9 are triggered by pathogen-specific nucleic acids (10-12). While mtDNA shares unmethylated $\mathrm{CpG}$ DNA repeats with bacterial DNA, mtDNA is also a ligand for TLR9 $(13,14)$. CpG DNA directly binds to TLR9 and induces TLR9 translocation (with alteration) from the endoplasmic reticulum to early endosomes and the later lysosomal compartment (15). Intracellular adapter protein myeloid differentiation factor-88 (MyD88) associated with TLR9 activates downstream signalling proteins, such as 
the interleukin-1 receptor-associated kinase (IRAK) family, mitogen-activated kinases (MAPK), or interferon-regulatory factors (IRFs) $(16,17)$.

Previous studies have indicated that TLR9 is also widely expressed in various human cancers, including breast, ovarian, prostate, brain, gastric, renal cell carcinoma, and oesophageal tumours (18-21). These data indicated that TLR9 serves a role as a prognosis marker as well as a biomarker for cancer diagnosis. A CpG island-containing oligodeoxyribonucleotide (ODN) called ODN-M362 is a synthetic CpG-rich sequence that contains unmethylated motifs, which is used for effective triggering of the TLR9-ligand binding-related events, which also mimics mtDNA function (22). Several preclinical findings have demonstrated the anticancer activity of $\mathrm{CpG}$ ODNs, which have been developed into TLR9-based agonist treatment $(22,23)$. In the present study, we quantified serum mtDNA levels in healthy subjects and NSCLC patients. It was found that lung cancer patients without metastasis had more mtDNA in serum as compared to the patients with metastasis. Moreover, TLR9-associated signalling was demonstrated after treatment with ODN-M362. In A549 and HCC827 cell lines, TLR9 signalling was activated and in addition, its adaptor protein, MyD88, was induced by ODN-M362 in a dose-dependent manner. This finding indicated that TLR9 may serve as a serological marker for NSCLC identification. Furthermore, we employed a human cytokine array to evaluate ODN-M362 stimulation of cytokines secretion. Hopefully, our findings may identify the role that TLR9 and mtDNA play in lung cancer progression and metastasis.

\section{Materials and methods}

Cell culture. A549 and HCC827 cells were cultured with F12K medium and RPMI-1640 (both from Sigma-Aldrich; Merck KGaA, Darmstadt, Germany) at $37^{\circ} \mathrm{C}$ in a humidified atmosphere $\left(95 \%\right.$ air, $\left.5 \% \mathrm{CO}_{2}\right)$, respectively. Fetal bovine serum (FBS; 10\%) (JRH Biosciences, Lenexa, KS, USA), $100 \mu \mathrm{g} / \mathrm{ml}$ penicillin/streptomycin and $2 \mathrm{mM}$ L-glutamine (Gibco; Thermo Fisher Scientific, Inc., Waltham, MA, USA) were supplemented in culture medium.

Subjects. Study subjects were recruited during routine health examinations with signed consents. Twenty-nine study subjects with varying age and sex were randomly chosen including 16 lung adenocarcinoma patients and 13 healthy volunteers, enrolling for analysis. This study was a prospective study that aimed to identify potential biomarkers to predict cancer progression. The Ethics Committee of the Taipei Tzu-Chi General Hospital approved the study (Approval no. TCRD-TPE-100-43; IRB no. 98-IRB-019-X; IRB extension no. 05-XD55-107). Our study was conducted from January 2010-January 2012 and December 2017-July 2018. The inclusion criteria for lung adenocarcinoma patients were as follows: The patients were required to be $>18$ years of age with the pathology type confirmed by pathology or cytology. Patients were excluded from the study if they had received chronic steroid therapy, adjuvant or neoadjuvant chemotherapy, radiotherapy, or both. Patients with a history of any other type of cancer were not eligible. The inclusion criteria for healthy volunteers were as follows: $>18$ years of age was required with no evidence of cancers. Healthy volunteers were excluded if they had inflammatory or infectious conditions (empyema, interstitial lung disease, or active pneumonitis) or a history of any other type of cancer.

Sample preparation and DNA extraction. Blood samples were centrifuged at $200 \mathrm{xg}$ at $8^{\circ} \mathrm{C}$ for $45 \mathrm{~min}$. Serum was transferred into new polypropylene tubes and re-centrifuged at $8,000 \mathrm{x} \mathrm{g}$ for $20 \mathrm{~min}$. The supernatant was collected and stored at $-80^{\circ} \mathrm{C}$. Serum DNA was extracted from $400 \mu 1$ sample with QIAmp DNA Blood Mini Kit (Qiagen, Inc., Valencia, CA, USA).

Quantitative PCR. DNA from patient serum was collected to performed quantitative (qPCR) on Applied Biosystems 7900 Sequence Detector (Applied Biosystems; Thermo Fisher Scientific, Inc.). The TaqMan probe sequence purchased from Applied Biosystems (Thermo Fisher Scientific, Inc.) was Mit 3153T (5'-FAM-TTCACAAAGCGCCTTCCCCCGTAA ATGA-TAMRA-3'), where FAM was 6-carboxyfluorescein and TAMRA was 6-carboxytetramethylrhodamine. The thermocycling conditions included an initial denaturation at $50^{\circ} \mathrm{C}$ for $2 \mathrm{~min}$ and $95^{\circ} \mathrm{C}$ for $10 \mathrm{~min}$, followed by 45 cycles at $95^{\circ} \mathrm{C}$ for $15 \mathrm{sec}, 60^{\circ} \mathrm{C}$ for $1 \mathrm{~min}$. The internal control primer sequences were plasmid forward primer (pls F) (5'-AATACG CAAACCGCCTCTCC-3') and plasmid reverse primer (pls R) (5'-ACAACATACGAGCCGGAAGC-3'). The internal control for the TaqMan probe sequence was plasmid TaqMan probe (pls T) (5'-FAM-CGCAACGCAATTAATGTGAGTTAG CTCAC-TAMRA-3'). The method of quantification used was $2^{-\Delta \Delta \mathrm{Cq}}(24)$.

Mitochondrial DNA copy number calibration. A 172-bp mtDNA from genomic DNA of a healthy volunteer was cloned and used as a calibrator. Calibrators were prepared by serial dilution of the stock solution and contained $10-10^{7}$ mitochondrial DNA copies $/ \mu$ l. The results were expressed as genome-equivalent $(\mathrm{GE}) / \mathrm{ml}$ of serum using a conversion factor of $6.6 \mathrm{pg}$ of DNA/cell. The concentration was calculated using an equation that was previously described (25). Primers used to amplify Mt3130-3301 were Mit3130F (5'-AGGACA AGAGAAATAAGGCC-3') and Mit 3301R (5'-TAAGAAGAG GAATTGAACCTCTGACTGT AA-3').

Western blotting. Cells were washed twice with ice-cold phosphate-buffered saline (PBS) (Gibco; Thermo Fisher Scientific, Inc., Waltham, MA, USA), lysed in $1 \mathrm{ml} /$ plate with ice-cold IP lysis buffer supplemented with protease inhibitors (Gibco; Thermo Fisher Scientific, Inc.) and harvested by scraping. Protein concentration was determined by BCA method. A total of $30 \mu \mathrm{g}$ of protein sample was separated by $10 \%$ SDS-PAGE, and then transferred onto polyvinylidene fluoride (PVDF) membranes (Bio-Rad Laboratories, Inc., Hercules, CA, USA). Membrane was blocked by 5\% non-fat dry milk in TBST with $0.05 \%$ Tween-20 for $1 \mathrm{~h}$. Primary antibodies to TLR9 (cat. no. ab88101; Abcam, Cambridge, MA, USA), MyD88 (cat. no. GTX112986; GeneTex International Corporation, Hsinchu, Taiwan) and $\beta$-tubulin (cat. no. sc-73242; Santa Cruz Biotechnology, Dallas, TX, USA) were used at a dilution of 1:1,000 and incubated at $4^{\circ} \mathrm{C}$ for overnight. Secondary antibodies to anti-mouse-HRP and anti-rabbit-HRP 
(cat. no. 31430 and 31460, Invitrogen; Thermo Fisher Scientific, Inc.) were used at a dilution of 1:5,000 and incubated at room temperature for $1 \mathrm{~h}$. Signals were detected with chemiluminescence (Amersham; GE Healthcare, Chicago, IL, USA) and exposed to X-ray film (Kodak, Rochester, NY, USA). Signal quantification was done by using ImageJ.

Cytokine array analysis. Culture medium (400 $\mu \mathrm{l})$ was harvested after cells were treated with CpG ODN-M362 for $24 \mathrm{~h}$ according to the manufacturer's instructions (Human Cytokine Array Panel A; R\&D Systems, Inc., Minneapolis, MN, USA). CpG-ODN-M362 sequence, 5'-TCGTCGTCG TTC:GAACGACGTTGAT-3'; and negative control, pCpG was a synthetic sequence called $\mathrm{pCpG}$ Giant that failed to trigger downstream TLR9 signaling and served as a negative control. pCpG Giant was purchased from InvivoGen (San Diego, CA, USA) (cat. no. tlrl-cpgg).

Statistical analysis. All experiments were repeated at least 3 times. Mean values and standard deviation (SD) were calculated and analyzed with a one-way analysis of variance (ANOVA) or Student's t-test with PRISM 5.0 (GraphPad Software, Inc., San Diego, CA, USA). Scheffe's post hoc test was used following one-way ANOVA. Data with $\mathrm{P}<0.05$ was considered to indicate a statistically significant difference.

\section{Results}

MtDNA is cloned from normal serum as a copy number calibrator. In order to evaluate the $\mathrm{CpG}$ level of different stages of tumorigenesis in lung cancer patients, a 172-bp mtDNA segment between nucleotide positions 3130 and 3301 was amplified from genomic DNA of a healthy volunteer with the primers. Mt3130-3301 was successfully amplified (Fig. 1A). The mtDNA amplicons were then cloned into the pCRII TOPO TA plasmid (Invitrogen; Thermo Fisher Scientific, Inc.) and were confirmed with restriction enzyme digestion and sequencing (Fig. 1B and $\mathrm{C}$ ). The cloned mtDNA plasmid was to be used as a calibrator for the mtDNA copy number.

To calibrate the correlation between threshold cycle $\left(\mathrm{C}_{\mathrm{T}}\right)$ in a TaqMan assay and the serum mtDNA copy number, a plot of the $\mathrm{C}_{\mathrm{T}}$ against the input target quantity, the plotted on a common log scale, was generated (Fig. 1D). The linearity of the quantitative assay was assessed with a calibrator, which was $172 \mathrm{bp}$ mtDNA flanked with a plasmid. The calibrator was serially diluted to concentrations from 10 to $10^{7} \mathrm{copies} / \mu \mathrm{l}$ before use. The mtDNA copy number was determined by division of the total DNA concentration by the weight of each plasmid molecule (25). The assay was sensitive and able to detect 10 copies of mitochondrial DNA/ $\mu 1$. As the plot indicated, the threshold cycle $\left(\mathrm{C}_{\mathrm{T}}\right)$ was set to 15 and was proportional to the target copy number from 10 to $10^{7} \mathrm{mtDNA}$ copies.

Patients without metastasis have higher serum mitochondrial DNA levels. To investigate the mtDNA copy number at different malignancy stages of lung cancer, we performed quantitative PCR analysis to evaluate the levels of mtDNA. Total DNA extraction from 13 healthy volunteers, 5 patients with non-metastatic, and 11 patients with metastatic lung adenocarcinoma (clinical characteristics of healthy subjects
Table I. Clinical characteristics of healthy and lung adenocarcinoma patients.

\begin{tabular}{lc}
\hline Characteristics & $\begin{array}{c}\text { Mean, medium (range) } \\
\text { or number (n) }\end{array}$ \\
\hline Healthy & $\mathrm{n}=13$ \\
Sex & 4 males/9 females \\
Age & $48.2 / 49.0(34-69)$ \\
Non-metastatic & $\mathrm{n}=5$ \\
Sex & 2 males/2 females \\
Age & $59.0 / 60.0(49-68)$ \\
Pathological stage & Stage II \\
Metastatic & $\mathrm{n}=11$ \\
Sex & 2 males/7 females \\
Age & $63.0 / 63.5(57-67)$ \\
Pathological stage & Stage IV \\
(brain, bone, and lung metastasis) & \\
\hline
\end{tabular}

and lung adenocarcinoma patients are summarised in Table I) was conducted, and we determined the concentration of serum mtDNA. Among these patients, 11 had lung adenocarcinoma of stage IV with metastasis, and 5 had stage II lung adenocarcinoma without metastasis. Serum mtDNA levels of the M0 (non-metastatic) group were significantly higher than those of the Meta (metastatic) and HS (healthy volunteer) groups (Fig. 2A). In addition, the Meta group had the lowest serum mtDNA level that was about a half of that of the M0 group. The qPCR results were then used to calculate mtDNA copy numbers with the calibrator (Fig. 2B). The mtDNA copy number was significantly higher in the M0 group, and up to $4 \times 10^{4}, \sim 4$ times higher than that in the HS group, indicating an associtaion between serum mtDNA levels and metastasis status during lung carcinogenesis.

Synthetic CpG ODN, ODN-M362, stimulates TLR9 and downstream MyD88 expression. To confirm that the high copy number of mtDNA functionally affects its receptor, TLR9, two human lung adenocarcinoma cell lines were treated with different concentrations of a synthetic unmethylated $\mathrm{CpG}$ ODN, ODN-M362, which has been known to serve as an agonist of TLR9, thereby simulating to the role of mtDNA by triggering TLR9-mediated signalling. A549 cells were treated with 1 or $3 \mu \mathrm{M}$ ODN-M362 for $24 \mathrm{~h}$ before harvesting. The expression level of TLR9 in A549 was induced by ODN-M362, and the downstream adaptor protein, MyD88, was also upregulated (Fig. 3A). In addition, the HCC827 cell line was also treated with 1,3 , or $5 \mu \mathrm{M}$ ODN-M362 for $24 \mathrm{~h}$. Compared with the A549 cells, the HCC827 cell line is also a lung adenocarcinoma cell line however it carries an acquired mutation in the EGFR tyrosine kinase domain. TLR9 and MyD88 in HCC827 cells were significantly upregulated by ODN-M362 treatment in a dose-dependent manner (Fig. 4A). Quantitative analysis of the western blotting was carried out relative to untreated cells (Figs. 3B and 4B). Thus, ODN-M362 was able to stimulate the expression of TLR9 in both lung 

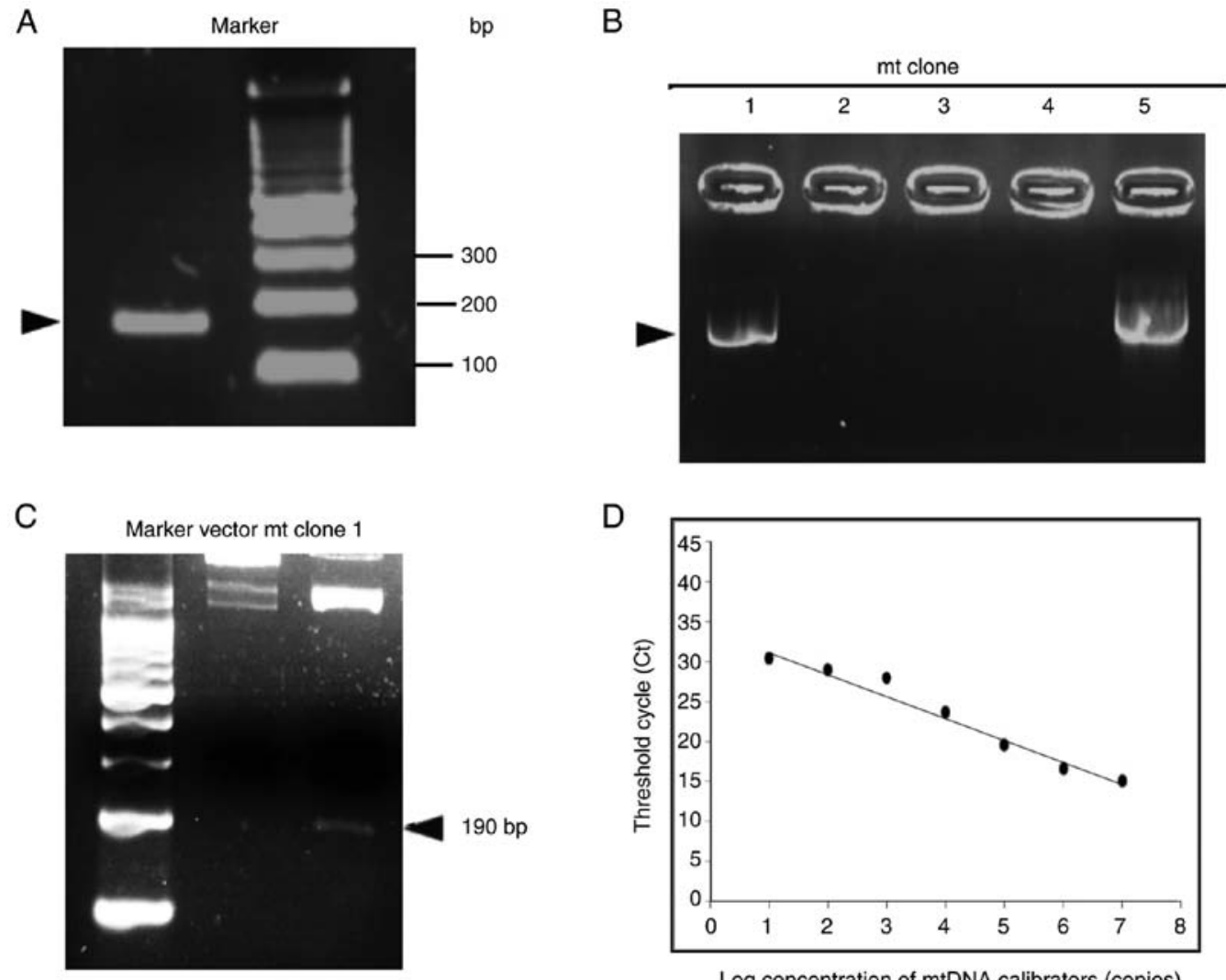

Log concentration of mtDNA calibrators (copies)

Figure 1. The Mit3130-Mit3301 fragment was cloned and is for calibration. (A) PCR was conducted to confirm the size of 172 bp mtDNA. (B) Eight single colonies were selected. Only colonies 1 (mt 1) and 5 (mt 5) exhibited insertions. (C) PCR results revealed a 190-bp product (arrow) after EcoRI digestion. (D) The threshold cycle (Ct) was the number of PCR cycles required for the fluorescent intensity of the reaction to reach a predefined threshold. The threshold cycle was inversely proportional to the logarithm of the starting concentration of mtDNA. mtDNA, mitochondrial DNA.

A

mtDNA in serum $\quad * * * P=0.00021$

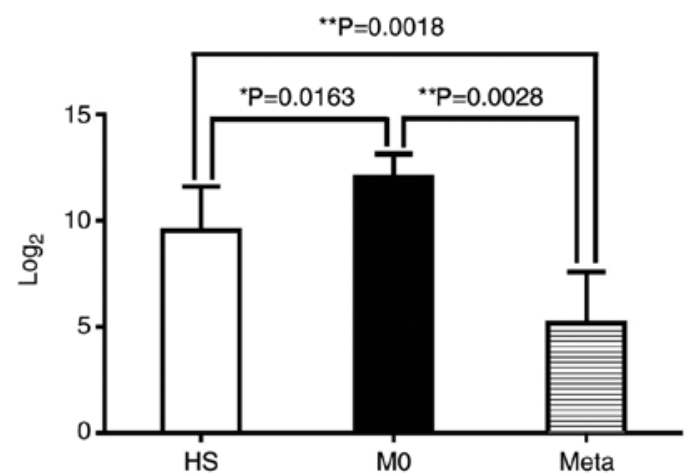

B

mtDNA in serum $\star * \star \mathrm{P}=0.00027$

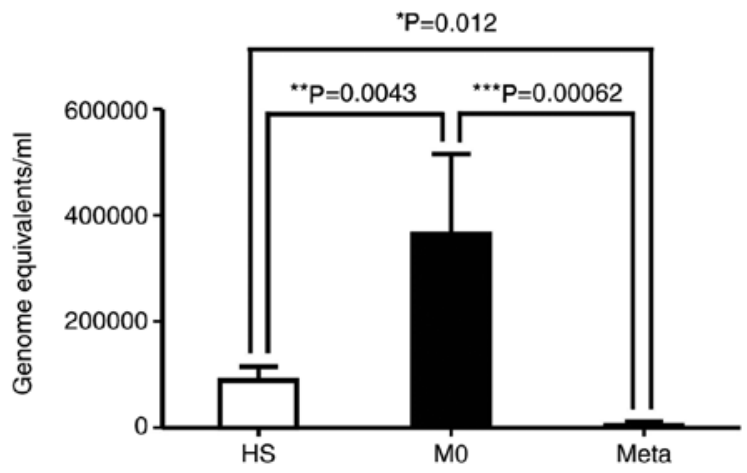

Figure 2. Quantitative PCR results reveal comparison of serum mtDNA levels between healthy volunteers and patients with lung adenocarcinoma with or without metastasis after calibration. (A) A $\log 2$ scale and (B) a genome-equivalent scale. (HS, healthy volunteer; M0, non-metastatic; Meta, metastatic). mtDNA, mitochondrial DNA. $\left({ }^{*} \mathrm{P}<0.1 ;{ }^{* *} \mathrm{P}<0.05 ;{ }^{* * *} \mathrm{P}<0.005\right)$.

cancer cell lines, and TLR9 may be regulated by mtDNA copy numbers in A549 and HCC827 cells.

Cytokine expression levels are altered by ODN-M362-mediated TLR9 signalling. We hypothesised that induction of TLR9 in lung adenocarcinoma cell lines activated downstream signalling through ODN-M362. Therefore, we performed a cytokine array analysis to assess the expression levels of 36 human cytokines with/without ODN-M362 treatment of A549 and HCC827 cell lines (Fig. 5A and C). Among the changes of cytokine expression, ODN-M362-treated A549 cells secreted IL- $8>15$-fold compared to the untreated cells, while C5/C5a was induced 1.6-fold in ODN-M362-treated A549 cells (Fig. 5B). It is known that $\mathrm{C} 5 / \mathrm{C} 5 \mathrm{a}$ is a direct inducer of interleukin-8 (IL-8) secretion (26). Therefore, marked increase of IL- 8 supports the previous finding and may be due to serial activation from the $\mathrm{C} 5 / \mathrm{C} 5 \mathrm{a}-$-mediated complement cascade. IL-8, an inflammation-associated chemokine, is 
A
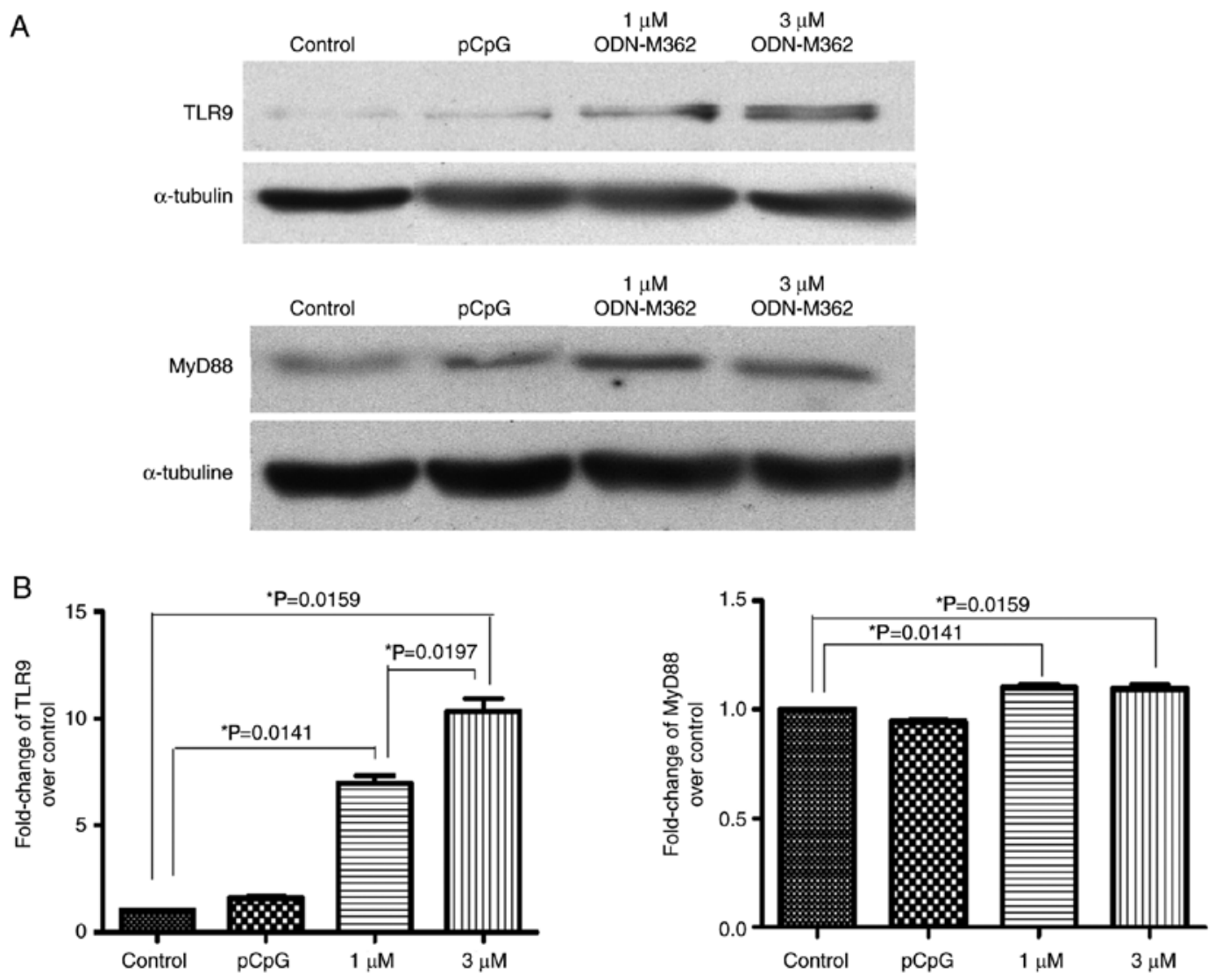

Figure 3. ODN-M362-treated A549 cells reveal induction of TLR9 and adaptor protein MyD88 expression. Western blotting was performed to determine the expression of TLR9 and MyD88. pCpG served as a negative control. (A) Dose-dependent TLR expression was detected as compared with a mock control, and expression was increased by ODN-M362. MyD88 expression was also increased by ODN-M362. (B) Quantitative analysis of A with P-values listed. ODN, oligodeoxyribonucleotide; MyD88, myeloid differentiation factor-88. $\left({ }^{*} \mathrm{P}<0.1\right)$

A
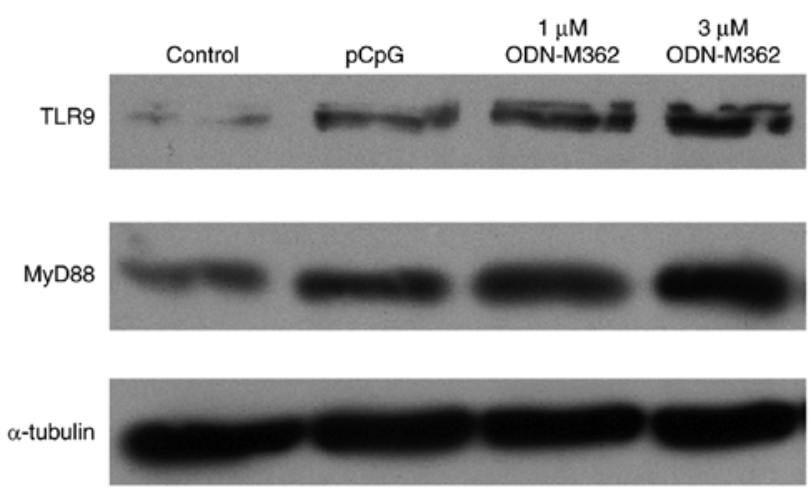

B
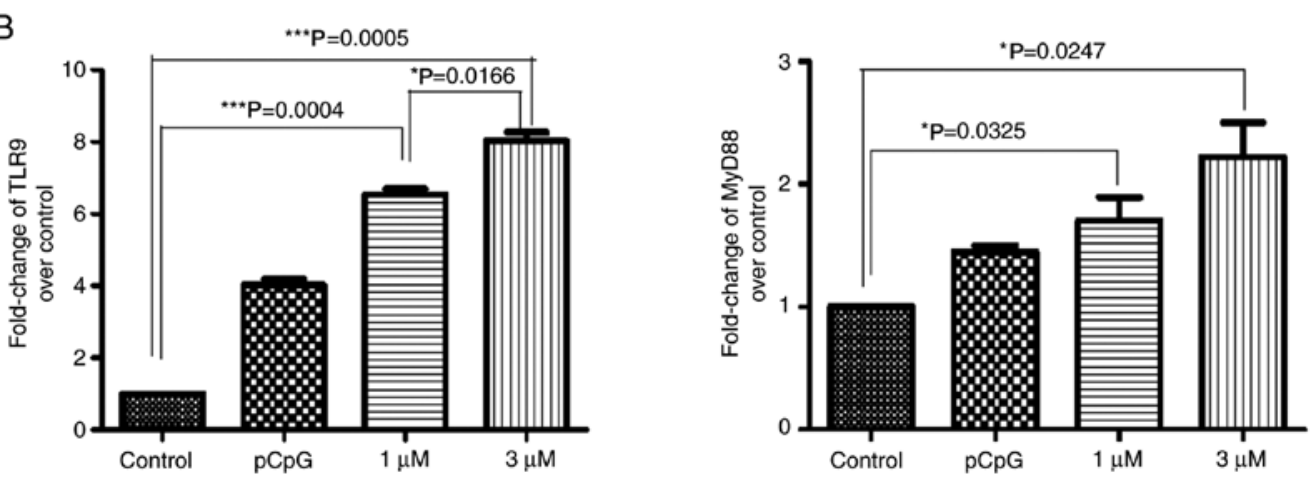

Figure 4. ODN-M362-treated HCC827 cells induced TLR9 and adaptor protein MyD88 expression. Western blotting was performed to determine the expression. pCpG served as a negative control. (A) Dose-dependent TLR expression was detected as compared with a mock control, and this expression was increased by ODN-M362. MyD88 expression was also increased by ODN-M362. (B) Quantitative analysis of (A) with P-value listed. ODN, oligodeoxyribonucleotide; MyD88, myeloid differentiation factor-88. $\left({ }^{*} \mathrm{P}<0.1 ;{ }^{* * * *} \mathrm{P}<0.005\right)$. 

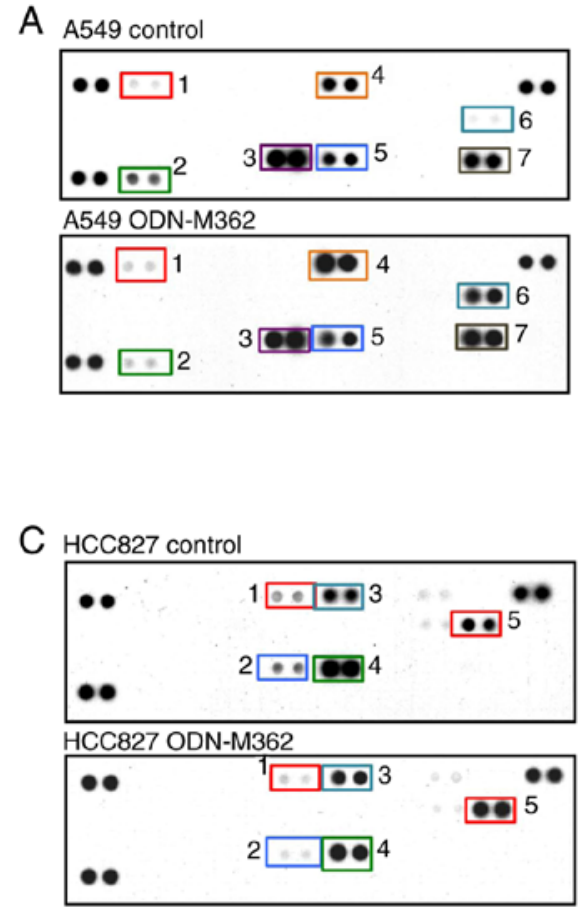

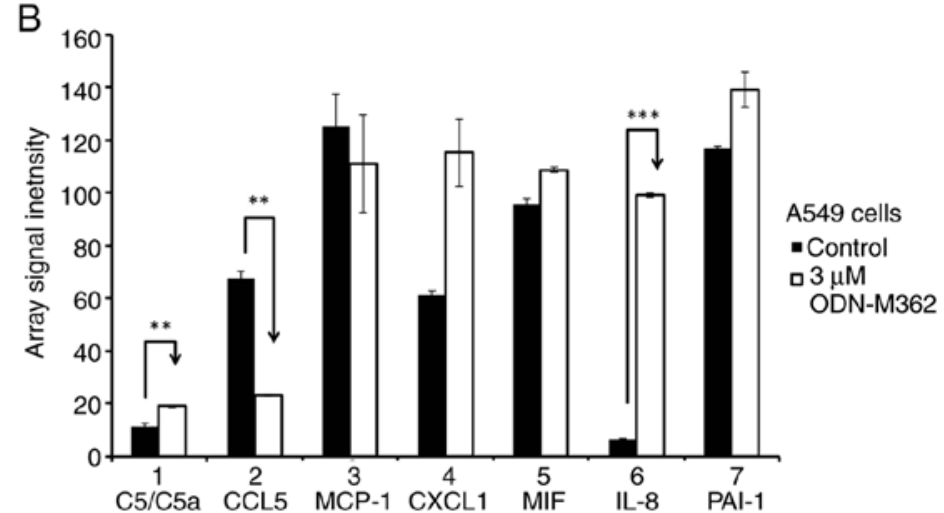

D

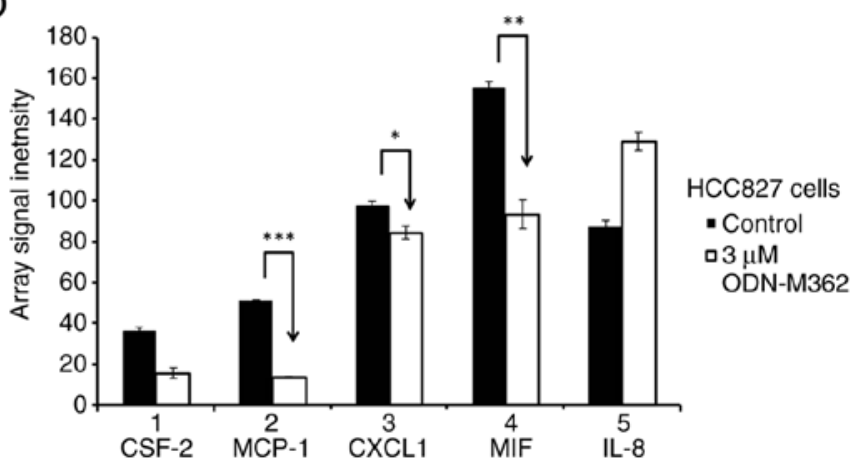

Figure 5. Human cytokine array analysis was performed on culture supernatants of A549 and HCC827 cells as compared with the control group or the $3 \mu \mathrm{M}$ ODN-M362-treated group. (A and B) C5/C5a and IL-8 levels were significantly elevated, but CCL5 expression was lower in ODN-M362-treated A549 cells. MCP-1 expression was diminished in ODN-M362-treated HCC827 cells but upregulated in ODN-M362-treated A549 cells. (C and D) MCP-1,S CXCL1, and MIF were downregulated in ODN-M362-treated HCC827 cells $\left({ }^{*} \mathrm{P}<0.1 ;{ }^{* *} \mathrm{P}<0.05 ;{ }^{* * *} \mathrm{P}<0.005\right)$. ODN, oligodeoxyribonucleotide.

also likely involved in cancer-associated processes, such as angiogenesis, proliferation, migration, and invasion (27). Moreover, IL-8 has been identified as an immunosuppressive cytokine that promotes carcinogenesis (28). Therefore, the ODN-M362-mediated induction of TLR9 signalling may cause inflammation-induced carcinogenesis through the IL-8 abnormality. Conversely, CCL5 was downregulated in ODN-M362-treated A549 cells (Fig. 5B). Although CCL5 has been reported as a protein that promotes cancer cell proliferation and migration (29), CCL5 also induces proper immune responses to tumour formation, and the mechanism is still unclear (28).

Both A549 and HCC827 are NSCLC cell lines; however, HCC827 cells are EGFR-mutated and highly sensitive to EGFR inhibitors with deletions from E746 to A750, whereas A549 cells encode wild-type EGFR, and these cell lines may induce different immune responses $(2,30)$. We found that after HCC827 cells were treated with ODN-M362, monocyte chemoattractant protein-1 (MCP-1), CXCL1, and macrophage migration inhibitory factor (MIF) were significantly downregulated (Fig. 5B and D). These results indicated that impaired EGFR signalling not only affects TLR9-mediated cytokine expression levels but also fails to induce chemotaxis for monocytes, neutrophils, and macrophages when TLR9 signalling is activated.

\section{Discussion}

It has been well established that mtDNA is involved in immune responses via TLR signalling. However, the precise role of mtDNA-mediated TLR signalling in different stages of human lung cancer has remained unclear. In the present study, the expression levels of serum mtDNA of healthy people and patients with NSCLC with or without metastasis were quantified. High levels of serum mtDNA in patients with non-metastatic lung adenocarcinoma as compared to healthy volunteers were determined; in contrast, NSCLC patients with metastasis displayed the lowest level of serum mtDNA. Although it has been confirmed that the copy number of mtDNA in gastric cancer is higher than that in benign tissue, the correlation between the copy number of mtDNA and clinical characteristics of cancer progression is still ambiguous (31). In spite of the controversy regarding the status of a prognostic biomarker, if we take the tumour node metastasis stage and the mtDNA copy number into consideration, there is still a significant synergistic effect on prognosis of colorectal cancer (32). In the present study, it was concluded that the serum mtDNA level is lower in patients with metastatic lung cancer, in agreement with a previous study on breast cancer (33). Although 3 years were spent collecting samples from NSCLC patients with or without metastasis; it was generally found that patients had less awareness before cancer metastasis to seek diagnosis, particularly for lung cancer. Therefore, this increased our challenge in obtaining enough samples for NSCLC without metastasis (M0). Nonetheless, according to the association between serum mtDNA copy number and metastasis status, the potential of detection of mtDNA levels in patients with or without metastasis as a pre-clinical diagnosis for identification of cancer progression is suggested. 
Next, a TLR9 agonist, ODN-M362 was used, to mimic the serum mtDNA effect and examine the expression level of TLR9 and its downstream targets in two different human lung cancer cell lines. It was observed that the expression of TLR9 was upregulated in both A549 and HCC827 cells after ODN-M362 treatment. Nonetheless, one of the downstream targets of TLR9 signalling, MyD88, was more significantly induced in HCC827 cells. While A549 is an EGFR wild-type cell line, HCC827 cells carry an oncogenic EGFR exon 19 deletion, rendering these cells sensitive to EGFR tyrosine kinase inhibitors (TKIs) (30). This finding indicated that ODN-M362 triggered much more effective TLR9 signalling in EGFR-mutated cells.

In order to clarify the effects of mtDNA-induced TLR9 signalling, we performed cytokine array analysis to identify the potential responsive elements. In A549 cells, activated TLR9 expression led to cancer progression and metastasis by increasing secretion of C5/C5a, IL-8, and CXCL1. When A549 cells were treated with ODN-M362, they may have transformed into cells with inflammation-induced malignancy through IL-8 aberrancy. In parallel, increasing TLR9 expression in HCC827 cells downregulated MCP-1, CXCL1, MIF and CSF-2. CpG DNAs have long been recognised as a therapeutic strategy with an immunostimulatory antitumour activity (22). However, the antitumour characteristic of ODN-M362 may be diminished by cell properties. When ODN-M362 was introduced into HCC827 cells, TLR9 signalling was increased, which revealed an mtDNA expression phenomenon similar to that in non-metastatic cancer patients. This result was consistent with the findings that patients with colorectal cancer had higher mtDNA expression and exhibited higher expression of immunosuppressive cytokines, such as IL-2 and TGF- $\beta 1$ (32).

Therefore, we deduced that the antitumour characteristic of ODN-M362 may have an improved therapeutic effect in NSCLC patients without metastasis. Moreover, HCC827 cells had more in common in terms of biological features with NSCLC patients without metastasis, and may provide a model for mtDNA-related research in the future. In addition, the cytokine expression diversity in A549 and HCC827 cell lines may serve as a referral index for identification of the corresponding cancer stages.

As for A549 and HCC827 cells, these two cell lines exhibited innate discrepancies in drug responses, including gefitinib and erlotinib. Although many well-known TKIs have been investigated regarding NSCLC treatment, EGFR appears to be the key element that determines drug sensitivity (34). Therefore, it is believed that EGFR mutation is the pivot that causes differential responses in A549 and HCC827 cells to ODN-M362 treatment; however, other factors may be involved and need to be clarified in the future.

In line with a previous finding concerning EGFR mutations associated with NSCLC, one upstream protein, CMTM7 was found to be responsible for a reduction in EGFR/AKT signalling in the HCC827 cell line. In addition, when the expression of CMTM7 was knocked down, it promoted NSCLC cell proliferation and metastasis (35). This result not only indicated that the intrinsic characteristic of HCC827 cells is less or no metastasis, but also indicated that the application of ODN-M362 to NSCLC therapy holds promise.
The ODN-M362-based therapy has been applied to numerous cancers, particularly lung cancer. It was proposed that TLR9 expression in malignant tumour cells may affect treatment approaches using a TLR9 agonist, such as CpG ODN. Combined with our findings, these data indicated that the NSCLC patients without metastasis have more serum mtDNA. This notion indicated that the endogenous high mtDNA level revealed suppression of tumour metastasis. Therefore, we concluded that serological mtDNA testing may serve as a diagnostic marker, and ODN-M362 therapy may be more effective before metastasis (36).

One research group reported that a novel immunomodulatory TLR9 agonist (IMO) inhibited the expression of EGFR, pMAPK, pAkt, TNF- $\alpha$, and Bcl-2 and had antitumour effects on human colon cancer xenografts. In addition, combined with an EGFR TKI, IMO-mediated inhibition of EGFR signalling was synergistically enhanced (37). More than $40 \%$ of lung cancers exhibit EGFR overexpression or mutations, and EGFR-targeted treatment has become one of the most common molecularly targeted therapies for lung cancer (38). In conclusion, although ODN-M362 caused significant induction of TLR9 signalling in the HCC827 cell line, we hypothesised that TLR9 agonists may fail to induce chemotaxis for leukocytes and may have a potential immunotherapeutic value in EGFR-mutated human lung adenocarcinoma. However, the biological role of TLR9 expression activated by ODN-M362 in advanced cancer and metastasis is still unclear and needs to be further evaluated.

In conclusion, serum mtDNA may serve as a diagnostic biomarker for identifying NSCLC with or without metastasis. Moreover, the different expression of TLR9-induced cytokines could also be an index for stage classification and may help diagnose progression and provide a prognosis in human lung cancer.

\section{Acknowledgements}

The authors thank the technical support from the Taipei Tzu Chi Hospital, Core Laboratory.

\section{Funding}

The present study was supported by the Buddhist Tzu Chi General Hospital grants: TCRD-TPE-95-25, TCRD-TPE-96-29 and TCRD-TPE-97-13 to SW.

\section{Availability of data and materials}

The datasets used during the present study are available from the corresponding author upon reasonable request.

\section{Authors' contributions}

SW and YPC conceived and designed the study. HYL, SW, CYH and YHL performed the experiments. YHL and SW wrote the paper. CYH, YHL and SW reviewed and edited the manuscript. All authors read and approved the manuscript and agree to be accountable for all aspects of the research in ensuring that the accuracy or integrity of any part of the work are appropriately investigated and resolved. 


\section{Ethics approval and consent to participate}

The Ethics Committee of the Taipei Tzu-Chi General Hospital approved the study (Approval no. TCRD-TPE-100-43; IRB no. 98-IRB-019-X; IRB extension no. 05-XD55-107). Patient written informed consent was obtained.

\section{Patient consent for publication}

Not applicable.

\section{Competing interests}

The authors declare that they have no competing interests.

\section{References}

1. Wallace DC: Mitochondrial DNA sequence variation in human evolution and disease. Proc Natl Acad Sci USA 91: 8739-8746, 1994.

2. Liu B, Du Q, Chen L, Fu G, Li S, Fu L, Zhang X, Ma C and Bin C: CpG methylation patterns of human mitochondrial DNA. Sci Rep 6: 23421, 2016.

3. Michl L and Shumacher HB Jr: Pulmonary valvular incompetence in growing animals. Surgery 73: 412-415, 1973.

4. Cavalli LR and Liang BC: Mutagenesis, tumorigenicity, and apoptosis: Are the mitochondria involved? Mutat Res 398: 19-26, 1998.

5. Parrella P, Xiao Y, Fliss M, Sanchez-Cespedes M, Mazzarelli P, Rinaldi M, Nicol T, Gabrielson E, Cuomo C, Cohen D, et al: Detection of mitochondrial DNA mutations in primary breast cancer and fine-needle aspirates. Cancer Res 61: 7623-7626, 2001.

6. Hosgood HD III, Liu CS, Rothman N, Weinstein SJ, Bonner MR, Shen M, Lim U, Virtamo J, Cheng WL, Albanes D, et al: Mitochondrial DNA copy number and lung cancer risk in a prospective cohort study. Carcinogenesis 31: 847-849, 2010.

7. Jakupciak JP, Maragh S, Markowitz ME, Greenberg AK, Hoque MO, Maitra A, Barker PE, Wagner PD, Rom WN, Srivastava S, et al: Performance of mitochondrial DNA mutations detecting early stage cancer. BMC Cancer 8: 285, 2008.

8. Galluzzi L, Kepp O and Kroemer G: Mitochondria: Master regulators of danger signalling. Nat Rev Mol Cell Biol 13: 780-788, 2012.

9. Goulopoulou S, Matsumoto T, Bomfim GF and Webb RC: Toll-like receptor 9 activation: A novel mechanism linking placenta-derived mitochondrial DNA and vascular dysfunction in pre-eclampsia. Clin Sci 123: 429-435, 2012.

10. Underhill DM, Ozinsky A, Smith KD and Aderem A: Toll-like receptor-2 mediates mycobacteria-induced proinflammatory signaling in macrophages. Proc Natl Acad Sci USA 96: 14459-14463, 1999.

11. Latz E, Visintin A, Espevik T and Golenbock DT: Mechanisms of TLR9 activation. J Endotoxin Res 10: 406-412, 2004.

12. Nishiya T, Kajita E, Miwa S and Defranco AL: TLR3 and TLR7 are targeted to the same intracellular compartments by distinct regulatory elements. J Biol Chem 280: 37107-37117, 2005.

13. Zhang Q, Raoof M, Chen Y, Sumi Y, Sursal T, Junger W, Brohi K, Itagaki K and Hauser CJ: Circulating mitochondrial DAMPs cause inflammatory responses to injury. Nature 464: 104-107, 2010.

14. Hemmi H, Takeuchi O, Kawai T, Kaisho T, Sato S, Sanjo H, Matsumoto M, Hoshino K, Wagner H, Takeda K, et al: A Toll-like receptor recognizes bacterial DNA. Nature 408: 740-745, 2000.

15. Latz E, Schoenemeyer A, Visintin A, Fitzgerald KA, Monks BG Knetter CF, Lien E, Nilsen NJ, Espevik T and Golenbock DT: TLR9 signals after translocating from the ER to CpG DNA in the lysosome. Nat Immunol 5: 190-198, 2004.

16. Akira S and Hoshino K: Myeloid differentiation factor 88-dependent and -independent pathways in toll-like receptor signaling. J Infect Dis 187 (Suppl 2): S356-S363, 2003.

17. Vollmer J: TLR9 in health and disease. Int Rev Immunol 25: $155-181,2006$
18. Berger R, Fiegl H, Goebel G, Obexer P, Ausserlechner M, Doppler W, Hauser-Kronberger C, Reitsamer R, Egle D, Reimer D, et al: Toll-like receptor 9 expression in breast and ovarian cancer is associated with poorly differentiated tumors. Cancer Sci 101: 1059-1066, 2010.

19. Väisänen MR, Väisänen T, Jukkola-Vuorinen A, Vuopala KS, Desmond R, Selander KS and Vaarala MH: Expression of toll-like receptor-9 is increased in poorly differentiated prostate tumors. Prostate 70: 817-824, 2010.

20. Kauppila JH, Takala H, Selander KS, Lehenkari PP, Saarnio J and Karttunen TJ: Increased Toll-like receptor 9 expression indicates adverse prognosis in oesophageal adenocarcinoma. Histopathology 59: 643-649, 2011.

21. Sandholm J and Selander KS: Toll-like receptor 9 in breast cancer. Front Immunol 5: 330, 2014.

22. Krieg AM: Toll-like receptor 9 (TLR9) agonists in the treatment of cancer. Oncogene 27: 161-167, 2008.

23. Wooldridge JE and Weiner GJ: CpG DNA and cancer immunotherapy: Orchestrating the antitumor immune response. Curr Opin Oncol 15: 440-445, 2003.

24. Livak KJ and Schmittgen TD: Analysis of relative gene expression data using real-time quantitative PCR and the $2^{-\Delta \Delta C_{\mathrm{T}}}$ method. Methods 25: 402-408, 2001.

25. Lo YM, Tein MS, Lau TK, Haines CJ, Leung TN, Poon PM, Wainscoat JS, Johnson PJ, Chang AM and Hjelm NM: Quantitative analysis of fetal DNA in maternal plasma and serum: Implications for noninvasive prenatal diagnosis. Am J Hum Genet 62: 768-775, 1998.

26. Vecchiarelli A, Retini C, Casadevall A, Monari C, Pietrella D and Kozel TR: Involvement of C3a and C5a in interleukin-8 secretion by human polymorphonuclear cells in response to capsular material of Cryptococcus neoformans. Infect Immun 66: 4324-4330, 1998.

27. Waugh DJ and Wilson C: The interleukin-8 pathway in cancer. Clin Cancer Res 14: 6735-6741, 2008.

28. Arango Duque G and Descoteaux A: Macrophage cytokines: Involvement in immunity and infectious diseases. Front Immunol 5: 491, 2014.

29. Aldinucci D and Colombatti A: The inflammatory chemokine CCL5 and cancer progression. Mediators Inflamm 2014: 292376, 2014.

30. Lee MS, Kim HP, Kim TY and Lee JW: Gefitinib resistance of cancer cells correlated with TM4SF5-mediated epithelial-mesenchymal transition. Biochim Biophys Acta 1823: 514-523, 2012.

31. Lee H, Lee JH, Kim DC, Hwang I, Kang YN, Gwon GJ, Choi IJ and Kim S: Is mitochondrial DNA copy number associated with clinical characteristics and prognosis in gastric cancer? Asian Pac J Cancer Prev 16: 87-90, 2015.

32. Qu F, Chen Y, Wang X, He X, Ren T, Huang Q, Zhang J, Liu X, Guo X, Gu J and Xing J: Leukocyte mitochondrial DNA content: A novel biomarker associated with prognosis and therapeutic outcome in colorectal cancer. Carcinogenesis 36: 543-552, 2015.

33. Weerts MJ, Sieuwerts AM, Smid M, Look MP, Foekens JA, Sleijfer S and Martens JW: Mitochondrial DNA content in breast cancer: Impact on in vitro and in vivo phenotype and patient prognosis. Oncotarget 7: 29166-29176, 2016.

34. Pao W and Chmielecki J: Rational, biologically based treatment of EGFR-mutant non-small-cell lung cancer. Nat Rev Cancer 10: 760-774, 2010.

35. Liu B, Su Y, Li T, Yuan W, Mo X, Li H, He Q, Ma D and Han W: CMTM7 knockdown increases tumorigenicity of human non-small cell lung cancer cells and EGFR-AKT signaling by reducing Rab5 activation. Oncotarget 6: 41092-41107, 2015.

36. Droemann D, Albrecht D, Gerdes J, Ulmer AJ, Branscheid D, Vollmer E, Dalhoff K, Zabel P and Goldmann T: Human lung cancer cells express functionally active Toll-like receptor 9 . Respir Res 6: 1, 2005.

37. Damiano V, Caputo R, Bianco R, D'Armiento FP, Leonardi A, De Placido S, Bianco AR, Agrawal S, Ciardiello F and Tortora G: Novel toll-like receptor 9 agonist induces epidermal growth factor receptor (EGFR) inhibition and synergistic antitumor activity with EGFR inhibitors. Clin Cancer Res 12: 577-583, 2006.

38. Han R, Wang X, Zhong D, Zhao J, Chen Z, Sun L, Wang J and Zhang J: Molecular mechanism of erlotinib resistance in epidermal growth factor receptor mutant non-small cell lung cancer cell line H1650. Zhongguo Fei Ai Za Zhi 15: 689-693, 2012 (In Chinese). 